\title{
TOPSIS Approach for Solving Bi-Level Non-Linear Fractional MODM Problems
}

\author{
Mahmoud A. Abo - Sinna ${ }^{a *}$, Azza H. Amer ${ }^{b}$
}

a. Department of Engineering Mathematics, Faculty of Engineering, El-Menoufiya University, Shebin El-Kom, Egypt.

b. Department of Mathematics, Faculty of Science, Helwan University, Cairo, Egypt.

* corresponding author (mabosinna2000@yahoo.com)

\begin{abstract}
TOPSIS (technique for order preference similarity to ideal solution) is considered one of the known classical multiple criteria decision making (MCDM) methods to solve bi-level non-linear fractional multi-objective decision making (BL-NFMODM) problems, and in which the objective function at each level is considered nonlinear and maximization type fractional functions. The proposed approach presents the basic terminology of TOPSIS approach and the construction of membership function for the upper level decision variable vectors, the membership functions of the distance functions from the positive ideal solution (PIS) and of the distance functions from the negative ideal solution (NIS). Thereafter a fuzzy goal programming model is adopted to obtain compromise optimal solution of BL-NFMODM problems. The proposed approach avoids the decision deadlock situations in decision making process and possibility of rejecting the solution again and again by lower level decision makers. The presented TOPSIS technique for BLNFMODM problems is a new fuzzy extension form of TOPSIS approach suggested by Baky and Abo-Sinna (2013) (Applied Mathematical Modelling, 37, 1004-1015, 2013) which dealt with bi -level multi-objective decision making (BL-MODM) problems. Also, an algorithm is presented of the new fuzzy TOPSIS approach for solving BL-NFMODM problems. Finally, an illustrative numerical example is given to demonstrate the approach.
\end{abstract}

Keyword: Multi-objective decision making, Bi-level programming problems, Fractional programming, TOPSIS, Fuzzy goal programming.

\section{Introduction}

Technique for order preference by similarity to ideal solution (TOPSIS), one of the known classical multiple criteria decision making (MCDM) methods, based upon the concept that the chosen alternative should have the shortest distance from the positive ideal solution (PIS) and the farthest from the negative ideal solution (NIS). It was first developed by Hwang and Yoon [1] for solving a multiple attribute decision making problem. Generally, TOPSIS provides a broader principle of compromise for solving multiple criteria decision-making problems. It transfers $\mathrm{m}$ - objectives (criteria), which are conflicting and non-commensurable, into two objectives (the shortest distance from the PIS and the longest distance from the NIS). They are commensurable and most time conflicting. Then, the bi-objective problem can be solved by using membership functions of fuzzy set theory to represent the satisfaction level for both criteria and obtain TOPSIS's compromise solution by a second-order compromise. The max - min operator is then considered as a suitable one to resolve the conflict between the new criteria (the shortest distance from the PIS and the longest distance from the NIS) [2,3,4]. Hwang and Yoon [1] used both PIS and NIS to normalize the distance family and obtain the form of distance family equations. Lia et al. [5] extended the concept of TOPSIS to develop a methodology for solving multiple objective decision making (MODM) problems. A similar concept has also been pointed out by Zeleny [6]. Recently, Baky and Abo-Sinna [7] proposed a fuzzy TOPSIS algorithm to solve bi-level multi-objective decision making (BL-MODM) problems. Abo - Sinna [2] extended TOPSIS approach to solve multi-objective dynamics programming (MODP) problems. As he showed that using the fuzzy max-min operator with non-linear membership functions, the obtained solutions are always non-dominated by the original MODP problems. Further extension of TOPSIS for large scale multi-objective non-linear programming problems with block angular structure was presented by Abo-Sinna et al. in [3,4]. Deng et al. [8] formulated the inter-company comparison process as a multi-criteria analysis model, and presented an effective approach by modifying TOPSIS for solving such a problem. Chen [9] extended the concept of TOPSIS to develop a methodology for solving multi-person multi -criteria decision-making problems in a fuzzy environment and he defined the fuzzy positive ideal solution (FPIS) and the fuzzy negative ideal solution (FNIS).

Bi-level programming problems (BLPs) concern with decentralized programming problems with two decision makers (DMs) in bilevel where decisions have interacted with each other were studied. A bibliography of the related references on bi-level programming problems in both linear and non-linear cases, which is updated biannually, can be found in [10]. In brief, the basic concept of the bilevel programming problem (BLPP) is that the upper-level decision makers (ULDMs) set their goals and/or decision, and then ask subordinate levels of the hierarchy for their optima, calculated in isolation. The lower level decision maker's (LLDMs) are then submitted and modified by the ULDM in consideration of the overall benefit for the organization or hierarchy. This process continues until a satisfactory solution is reached. Bi - level organization has the following common characteristics: Interactive decision-making units exist within a predominantly hierarchical structure; the execution of decisions is sequential from upper-level to lower-level; each decision-making unit independently controls a set of decision variables and is interested in maximizing its own objective but is affected by the reaction of lower-level $\mathrm{DM}_{\mathrm{s}}$ due to their dissatisfaction with the decision of the upper- level DM. So, the decision deadlock arises frequently in the decision-making situation.

Over the last three decades, tremendous amount of research efforts has been made on multi-level programming problems (MLPP $)$ for hierarchical decentralized planning problems leading to the publication of many interesting results in literature $[11,12,13,14,15,16,17,18,19]$ and many methodologies have been proposed to solve MLPP's which potentially arise in various fields such as Agriculture, Bio fuel production, Economic systems, Finance Government policy, Network designs etc. Candler and Townsley [20] have suggested applications of multi-level programming in governmental problems involving issues such as the setting of penalties for illegal drug import, the fixing of import quotas and the development of transportation and communications infrastructure. Applications to strategic weapons exchange problems and to the distribution of federal budgets among states have been described 
respectively by Bracken et al. [21] and Cassidy et al. [22]. Anandilingam and Apprey [13] have given a new approach to conflict resolution based on multi-level mathematical programming and have illustrated it with a real-world example of the Ganga water conflict problem between India and Bangladesh.

In real world decision situations, decision makers may sometimes face up with the decision at different levels as: to optimize multiple objective functions like inventory, sales, actual cost, standard cost, output, employee, etc. with respect to some constraints. Such type of problems in a large hierarchical organization from upper-level to lower-level and their sequential decisions on complex and conflicting multiple objectives formulate the multi-level multi-objective decision making problems (ML- MODMs). In general, Real-world problems are characterized by the presence of many often conflicting and incommensurable objectives at different hierarchical levels defined as multi-level multi-objective decision making (ML-MODM) problems [23].

In recent years, some researchers have started discovering solution methodologies for complex hierarchical problems. Recently, Baky [24] suggested two new techniques with fuzzy goal programming (FGP) approach based on solution preferences by the decision maker at each level to solve new type of problem multi-level multi-objective linear programming (ML-MOLP) problems through FGP approach. Abo-Sinna and Baky [25] presented interactive balance space approach for solving multi- level multi-objective programming problems. Baky [26] proposed FGP algorithm for solving decentralized bi-level multi-objective programming (DBLMOP) problems with a single decision maker at the upper-level and multiple decision makers at the lower level. Lachhwani [27] suggested an alternate technique based on FGP for solving ML-MOLPP. Lachhwani [28] presented a new modified method for solving multi-level multi-objective linear fractional programming problems (ML-MOLFPP ${ }_{s}$ ) based on fuzzy goal programming (FGP) approach. Abo-Sinna and Baky [29] proposed interactive balance space approach for bi-level multi-objective programming problems.

Also, in [30,31,32,33,34,35,36] many researchers have designed algorithms for studying the bi-level quadratic fractional optimization problem through fuzzy goal programming methodology to find the optimal solution of it.

In this paper, we propose the new fuzzy TOPSIS (technique for order preference by similarity to ideal solution) approach to solve bi-level non- linear fractional multi-objective decision-making (BL-NFMODM) problems, and in which the objective function at each level are considered maximization type non-linear functions. The proposed approach presents the basic terminology of TOPSIS approach and the construction of membership function for the upper-level decision variable vectors, the membership functions of the distance functions from the positive ideal solution (PIS) and of the distance functions from the negative ideal solution (NIS) and there after fuzzy goal programming model is adopted to obtain compromise optimal solution of ML-NFMODM problems. The proposed approach avoids the decision deadlock situations in decision making process and possibility of rejecting the solution again and again by lower-level decision makers. The proposed TOPSIS technique for BL-NFMODM problems is an extension form of TOPSIS approach suggested by Baky and Abo-Sinna [7] which dealt with bi-level multi-objective decision making (BL-MODM) problems. An illustrative numerical example is given to demonstrate the approach.

\section{Quadratic Fractional Programming Problem (QFPP)}

Transformation QFPP to non-linear programming problem (NLPP), taking a simple model of QFPP [37, 38]:

$$
\operatorname{Min} Z=\frac{Q_{1} x_{1}^{2}+Q_{2} x_{2}^{2}+c_{1} x_{1}+c_{2} x_{2}+c_{3}}{R_{1} x_{1}^{2}+R_{2} x_{2}^{2}+d_{1} x_{1}+d_{2} x_{2}+d_{3}}
$$

Subject to $A_{1} X_{1}+A_{2} X_{2} \leq b$

Suppose that,

$$
\begin{gathered}
\frac{1}{R_{1} x_{1}^{2}+R_{2} x_{2}^{2}+d_{1} x_{1}+d_{2} x_{2}+d_{3}}=t^{2}, \\
y_{1}=t x_{1}, \text { and } y_{2}=t x_{2} . \\
Z=\left[Q_{1} \frac{y_{1}^{2}}{t^{2}}+Q_{2} \frac{y_{2}^{2}}{t^{2}}+c_{1} \frac{y_{1}}{t}+c_{2} \frac{y_{2}}{t}+c_{3}\right] t^{2} ; \text { or } \\
Z=Q y_{1}^{2}+Q_{1} y_{2}^{2}+c_{1} t y_{1}+c_{2} t y_{2}+c_{3} t^{2}
\end{gathered}
$$

So,

subject to $A_{1} x_{1}+A_{2} X_{2} \leq b$, becomes $A_{1} y_{1}+A_{2} y_{2}-b t \leq 0$,

Also, $\frac{1}{t^{2}}=R_{1} x_{1}^{2}+R_{2} x_{2}^{2}+d_{1} x_{1}+d_{2} x_{2}+d_{3}$ gives 


$$
\begin{aligned}
& \frac{1}{t^{2}}=R_{1} \frac{y_{1}^{2}}{t^{2}}+R_{2} \frac{y_{2}^{2}}{t^{2}}+d_{1} \frac{y_{1}}{t}+d_{2} \frac{y_{2}}{t}+d_{3} ; \text { or, } \\
& 1=R_{1} y_{1}^{2}+R_{2} y_{2}^{2}+d_{1} t y_{1}+d_{2} t y_{2}+d_{3} t^{2}
\end{aligned}
$$

Therefore problem (P-1) can be equivalently transformed as:

$$
\begin{gathered}
\operatorname{Min} Z=Q_{1} y_{1}^{2}+Q_{2} y_{2}^{2}+c_{1} t y_{1}+c_{2} t y_{2}+c_{3} t^{2} \\
\text { subject to } A_{1} y_{1}+A_{2} y_{2}-b t \leq 0, \\
R_{1} y_{1}^{2}+R_{2} y_{2}^{2}+d_{1} t y_{1}+d_{2} t y_{2}+d_{3} t^{2}=1, \\
y_{1} \geq 0, y_{2} \geq 0, t>0
\end{gathered}
$$

\section{Problem Formulation}

Assume that there are two levels in a hierarchy structure with upper-level decision maker (ULDM) and lower-level decision maker (LLDM). Let the vector of decision variables $x=\left(x_{1}, x_{2}\right) \in R^{n}$ be partitioned between the two decision makers. The upperlevel decision maker has control over the vector $x_{1} \in R^{n_{1}}$ and the lower-level decision maker has control over the vector $x_{2} \in R^{n_{2}}$, where $n=n_{1}+n_{2}$. Furthermore, assume that:

$$
F_{i}\left(x_{1}, x_{2}\right): R^{n_{1}} \times R^{n_{2}} \rightarrow R^{m_{i}}, i=1,2 .
$$

are the upper-level and lower-level vector of non-linear objective functions, respectively. So, the BL-NFMODM problem of maximization type may be formulated as follows:

[Upper Level]

$$
\underset{x_{1}}{\operatorname{Max}_{1}} F_{1}\left(x_{1}, x_{2}\right)=\operatorname{Max}_{x_{1}}\left(F_{11}\left(x_{1}, x_{2}\right), F_{12}\left(x_{1}, x_{2}\right), . . F_{1 m_{1}}\left(x_{1}, x_{2}\right)\right),
$$

where $x_{2}$ solves

[Lower Level]

$$
\underset{x_{2}}{\operatorname{Max}} F_{2}\left(x_{1}, x_{2}\right)=\underset{x_{2}}{\operatorname{Max}}\left(F_{21}\left(x_{1}, x_{2}\right), F_{22}\left(x_{1}, x_{2}\right), \ldots, F_{2 m_{2}}\left(x_{1}, x_{2}\right)\right),
$$

subject to

$$
x \in G=\left\{x=\left(x_{1}, x_{2}\right) \in R^{n} \mid g_{k}\left(x_{1}, x_{2}\right) \leq 0, k=1,2, \ldots, q\right\} \neq \phi
$$

where $F_{i j}\left(x_{1}, x_{2}\right)=\frac{f_{i j}\left(x_{1}, x_{2}\right)}{g_{i j}\left(x_{1}, x_{2}\right)}, i=1,2 ; j=1,2, \ldots, m_{i}$,

$f_{i j}\left(x_{1}, x_{2}\right)$ and $g_{i j}\left(x_{1}, x_{2}\right)$ are non- linear functions, $x_{1}=\left(x_{11}, x_{12}, \ldots, x_{1 n_{1}}\right)$,

$x_{2}=\left(x_{21}, x_{22}, \ldots, x_{2 n_{2}}\right), \mathrm{G}$ is the convex constraints feasible choice set, $m_{i}, i=1,2$ are the number of DMi's objective functions, and $\mathrm{q}$ is the number of the constraints.

Using the transformation method $\left(\mathrm{T}_{1}\right)$ proposed by Charnos and Cooper [37] and illustrated in detail in section 2, problem [(1-a)-(1-d)] is equivalent to the following BL-NMODM as:

[Upper Level] 


$$
\underset{y_{1}}{\operatorname{Max}_{1}} F_{1}^{\prime}(y, t)=\operatorname{Max}_{y_{1}}\left(f_{11}(y, t), f_{12}(y, t), \ldots, f_{1 m_{1}}(y, t)\right) \quad\left(1-a^{\prime}\right)
$$

where $y_{2}$ solves

[Lower Level]

$$
\underset{y_{2}}{\operatorname{Max}_{2}} F_{2}^{\prime}(y, t)=\underset{y_{2}}{\operatorname{Max}}\left(f_{21}(y, t), f_{22}(y, t), \ldots, f_{2 m_{2}}(y, t)\right) \quad\left(1-b^{\prime}\right)
$$

subject to

$$
(y, t) \in M=\left\{y=\left(y_{1}, y_{2}, t\right) \mid g_{l}^{\prime}(y, t) \leq 0, l=1,2, \ldots, q+m_{1}+m_{2}\right\} \neq \phi \quad\left(1-c^{\prime}\right)
$$

where $\mathrm{q}$ is the number of the constraints, $m_{1}$ is the upper-level number of $g_{1 m_{1}}$ and $m_{2}$ is the lower-level number of $g_{2 m_{2}}$.

\section{Some Basic Concepts of Distance Measures}

This section briefly surveys some basic concepts of distance measures, for more details see $[2,3,4,5,7,39,40]$. To obtain a compromise solution of MODM problems of the form:

$$
\begin{aligned}
& \operatorname{Max} F(x)=\left(f_{1}(x), f_{2}(x), \ldots, f_{m}(x)\right), \\
& x \in G=\left\{x \in R^{n} \mid g_{i}(x) \leq 0, i=1,2, \ldots, q\right\} \neq \phi .
\end{aligned}
$$

subject to

Consider the vector of objective functions $F(x)=\left(f_{1}(x), f_{2}(x), \ldots, f_{m}(x)\right)$ and the ideal vector of objective functions $F^{*}=\left(f_{1}^{*}, f_{2}^{*}, \ldots, f_{m}^{*}\right)$ (ideal point- reference point-positive ideal solution (PIS)) in the $m$-objective space. And consider the vector of anti-ideal solution of objective functions $F^{-}=\left(f_{1}^{-}, f_{2}^{-}, \ldots, f_{m}^{-}\right)$(anti-ideal point-nadir point-negative ideal solution (NIS)), where $f_{j}^{*}=\max _{x \in G} f_{j}(x)$ and $f_{j}^{-}=\min _{x \in G} f_{j}(x), j=1,2, \ldots, m$. As the measure of "closeness", $L_{p^{-}}$ metric is used, the $L_{p}$-metric defines the distance between two points $F(x)$ and $F^{*}$ as:

$$
d_{p}=\left\{\sum_{j=1}^{m} \lambda_{j}^{p}\left[f_{j}^{*}-f_{j}(x)\right]^{p}\right\}^{\frac{1}{p}}, p=1,2, \ldots, \infty,
$$

where $\lambda_{j}, j=1, \ldots, m$ are the relative importance (weights) of objectives. If the objective functions $f_{j}(x), j=1, \ldots, m$ are not expressed in commensurable units, then a scaling function for every objective functions, usually, this dimensionless is the interval $[0,1]$. In this case, the following metric could be used:

$$
d_{p}=\left\{\sum_{j=1}^{m} \lambda_{j}^{p}\left[\frac{f_{j}^{*}-f_{j}(x)}{f_{j}^{*}-f_{j}^{-}}\right]^{p}\right\}^{\frac{1}{p}}, p=1,2, \ldots, \infty .
$$

The global criterion method, goal programming, fuzzy programming, and interactive approaches use the distance family of (3) and (4) when the ideal vector of objective functions $F^{*}=\left(f_{1}^{*}, f_{2}^{*}, \ldots, f_{m}^{*}\right)$ being the reference point. The problem becomes how to solve the following auxiliary problem:

$$
\operatorname{Mind}_{x \in G}=\left\{\sum_{j=1}^{m} \lambda_{j}^{p}\left[\frac{f_{j}^{*}-f_{j}(x)}{f_{j}^{*}-f_{j}^{-}}\right]^{p}\right\}^{\frac{1}{p}}, p=1,2, \ldots, \infty .
$$


The value chosen for $p$ reflects the way of achieving a compromise by minimizing the weight sum of the divisions of objective from their respective reference point (ideal solution). The parameter $p$ plays the role of the "balancing factor" between the group utility and maximal individual regret. As $p$ increases, the group utility (distance $d_{p}$ ) decreases, i.e. $d_{1} \geq d_{2} \geq \ldots \geq d_{p}$ and greater emphasis is given to the largest deviation in forming the total. Specifically, $p=1$ implies an equal importance (weights) for all these deviations, while $p=2$ implies that these deviations are weighted proportionately with the largest deviation having the largest weight [5]. Finally for $p=\infty$, the largest deviation completely dominates the distance determination, the $L_{\infty}$ - metric is of the form:

$$
d_{\infty}=\operatorname{Max}_{j}\left\{\lambda_{j}\left[f_{j}^{*}-f_{j}(x)\right]\right\} \operatorname{or} d_{\infty}=\operatorname{Max}_{j}\left\{\lambda_{j}\left[\frac{f^{*}-f_{j}(x)}{f_{j}^{*}-f_{j}^{-}}\right]\right\} .
$$

\section{TOPSIS for BL-NMODM Problems}

In most practical situations, we might like to have a decision, which not only makes as much profit as possible, but also avoids as much risk as possible. This concept has been developed by Hwang and Yoon [1]. They provided a new approach, TOPSIS, for solving a multiple attribute decision making (MADM) problems. It is based upon the principle that the chosen alternative should have the shortest distance from the positive ideal solution (PIS) and the farthest from the negative ideal solution (NIS). Hwang and Yoon used both PIS $\left(F^{*}\right)$ and NIS $\left(F^{-}\right)$to normalize the distance family and obtain the form of distance family of Eq. (4). Lia et al.[5] extended the concept of TOPSIS to develop a methodology for solving multiple objective decision making (MODM) problems. In this paper, the researchers further extended the concept of TOPSIS [5] for BLNMODM problems.

\subsection{The TOPSIS Approach for the Upper NMODM Problem}

Consider the upper level multi-objective of maximization type problem of the BL-NMODM problem $\left(1^{\prime}\right)$ :

$$
\operatorname{Max}_{y_{1}} F_{1}^{\prime}(y, t)=\underset{y_{1}}{\operatorname{Max}}\left(f_{11}(y, t), f_{12}(y, t), \ldots, f_{1 m_{1}}(y, t)\right)
$$

subject to

$$
(y, t) \in M=\left\{\left(y_{1}, y_{2}, t\right) \mid g_{l}(y, t) \leq 0, l=1,2, \ldots, q+m_{1}+m_{2}\right\}
$$

The TOPSIS approach of Lia et al. [5] that solves single level MODM problems is considered. In this paper, to solve the upper-level NMO problem, the TOPSIS model formulation of this approach can be briefly stated as following, for more details see [5]:

$$
\begin{aligned}
& \operatorname{Mind}_{p}^{P I S^{u}}(y, t) \\
& \operatorname{Max}_{p}{ }^{N I S^{u}}(y, t)
\end{aligned}
$$

subject to

$$
(y, t) \in M=\left\{\left(y_{1}, y_{2}, t\right) \mid g_{l}(y, t) \leq 0, l=1,2, \ldots, q+m_{1}+m_{2}\right\}
$$

where

$$
d_{p}^{P I S^{u}}(y, t)=\left\{\sum_{j=1}^{m_{1}} \lambda_{j}^{p}\left[\frac{f_{1 j}^{*}-f_{1 j}(y, t)}{f_{1 j}^{*}-f_{1 j}^{-}}\right]^{p}\right\}^{\frac{1}{p}} \text { and } d_{p}^{N I S^{u}}(y, t)=\left\{\sum_{j=1}^{m_{1}} \lambda_{j}^{p}\left[\frac{f_{1 j}-f_{1 j}^{-}}{f_{1 j}^{*}-f_{1 j}^{-}}\right]^{p}\right\}^{\frac{1}{p}}
$$

and where $f_{1 j}^{*} f_{1 j}^{-}$and $\lambda_{j}, j=1,2, \ldots, m_{1}$ (the individual positive ideal solutions, the individual negative ideal solutions and the relative importance (weights) of objectives, respectively) are defined as in section 4 . Let $F^{u^{*}}=\left(f_{11}^{*}, f_{12}^{*}, \ldots f_{1 m_{1}}^{*}\right)$ and $F^{u^{-}}=\left(f_{11}^{*}, f_{12}^{*}, \ldots f_{1 m_{1}}^{*}\right)$. Assume that the membership functions $\left(\mu_{1}(y, t)\right.$ and $\left.\mu_{2}(y, t)\right)$ of the two objective functions are linear between $\left(d_{p}^{u}\right)^{*}$ and $\left(d_{p}^{u}\right)^{-}$which are: 


$$
\begin{aligned}
& \left(d_{p}^{P I S^{u}}\right)^{*}=\min _{M} d_{p}^{P I S^{u}}(y, t) \text { and the solution is }(y, t)^{p} \\
& \left(d_{p}^{N I S^{u}}\right)^{*}=\min _{M} d_{p}^{N I S^{u}}(y, t) \text { and the solution is }(y, t)^{N} \\
& \left(d_{p}^{P I S^{u}}\right)^{-}=d_{p}^{P I S^{u}}(y, t)^{N} \text { and }\left(d_{p}^{N I S^{u}}\right)^{-}=d_{p}^{N I S^{u}}(y, t)^{P}
\end{aligned}
$$

Also, we propose in this paper that, $\left(d_{p}^{P I S^{u}}\right)^{-}$and $\left(d_{p}^{N I S^{u}}\right)^{*}$ can be taken as $\left(d_{p}^{P I S^{u}}\right)^{-}=\max _{M} d_{p}^{P I S^{u}}(y, t)$ and $\left(d_{p}^{N I S^{u}}\right)^{*}=\max _{M} d_{p}^{N I S^{u}}(y, t)$, respectively.

$d_{p}^{u^{*}}=\left(\left(d_{p}^{P I S^{u}}\right)^{*},\left(d_{p}^{N I S^{u}}\right)^{*}\right)$ and $d_{p}^{u^{-}}=\left(\left(d_{p}^{P I S^{u}}\right)^{-},\left(d_{p}^{N I S^{u}}\right)^{-}\right)$. $\mu_{1}(y, t) \equiv \mu_{d_{p}^{P I S} S^{u}}(y, t)$ and $\mu_{2}(y, t) \equiv \mu_{d_{p}^{N I S}}(y, t)$ can be obtained as (see: Fig.1):

$\mu_{1}(y, t)= \begin{cases}1 \quad, \text { if } d_{p}^{P I S^{u}}(y, t)<\left(d_{p}^{P I S^{u}}\right)^{*}, \\ 1-\frac{d_{p}^{P I S^{u}}(y, t)-\left(d_{p}^{P I S^{u}}\right)^{*}}{\left(d_{p}^{P I S^{u}}\right)^{-}-\left(d_{p}^{P I S^{u}}\right)^{*}}, & \text { if }\left(d_{p}^{P I S^{u}}\right)^{*} \leq d_{p}^{P I S^{u}}(y, t) \leq\left(d_{p}^{P I S^{u}}\right)^{-} \\ 0 & , \text { if }\left(d_{p}^{P I S^{u}}\right)^{-}<d_{p}^{P I S^{u}}(y, t) .\end{cases}$

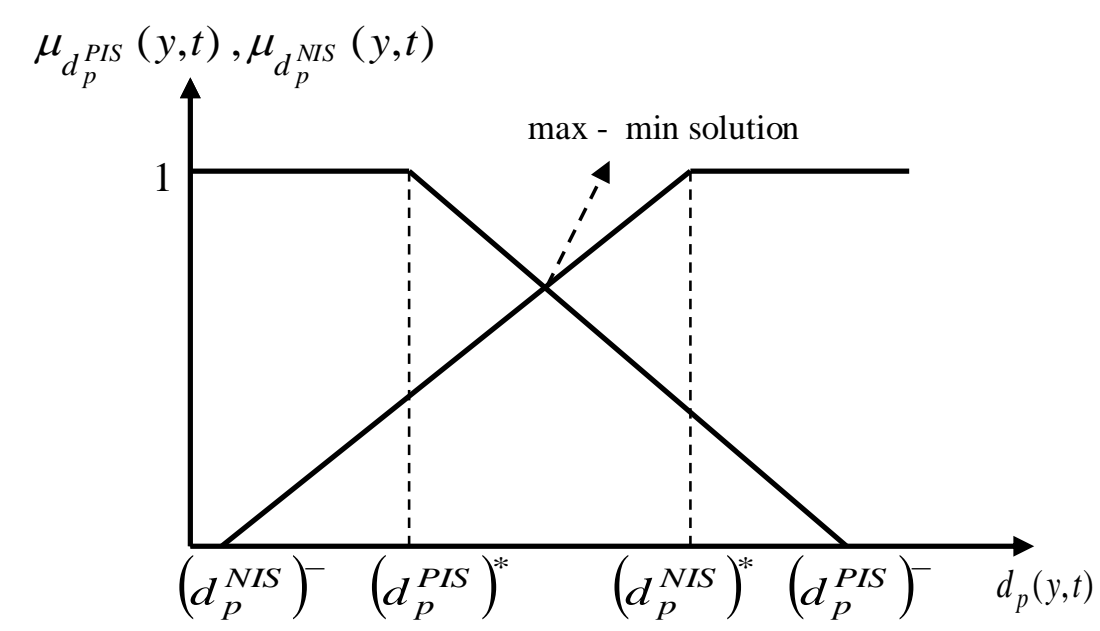

Fig. 1. The membership functions of $\mu_{d_{p}^{P I S}}(y, t)$ and $\mu_{d_{p}^{N I S}}(y, t)$ [5]. 


$$
\mu_{2}(y, t)= \begin{cases}1 & \text {,if } d_{p}^{N I S^{u}}(y, t) \geq\left(d_{p}^{N I S^{u}}\right)^{*}, \\ 1-\frac{\left(d_{p}^{N I S^{u}}\right)^{*}-d_{p}^{N I S^{u}}(y, t)}{\left(d_{p}^{N I S^{u}}\right)^{*}-\left(d_{p}^{N I S^{u}}\right)^{-}}, & \text {if }\left(d_{p}^{N I S^{u}}\right)^{-} \leq d_{p}^{N I S^{u}}(y, t) \leq\left(d_{p}^{N I S^{u}}\right)^{*}, \\ 0 & , \text { if } d_{p}^{N I S^{u}}(y, t)<\left(d_{p}^{N I S^{u}}\right)^{-} .\end{cases}
$$

Applying the max-min decision model, which is proposed by Bellmann and Zadeh [41] and extended by Zimmermann [42,43], we can resolve (8) and obtain the satisfying decision of upper-level NMODM problem, $(y, t)^{u^{*}}=\left(y_{1}^{u^{*}}, y_{2}^{u^{*}}\right)$, by solving the following problem:

$$
\mu_{D}(y, t)=\max _{M}\left\{\min \left(\mu_{1}(y, t), \mu_{2}(y, t)\right)\right\}
$$

where $(y, t)_{1}^{u^{*}}=\left((y, t)_{11}^{u^{*}},(y, t)_{12}^{u^{*}}, \ldots,(y, t)_{1 n_{1}}^{u^{*}}\right)$ and $(y, t)_{2}^{u^{*}}=\left((y, t)_{21}^{u^{*}},(y, t)_{22}^{u^{*}}, \ldots,(y, t)_{2 n_{2}}^{u^{*}}\right)$.

If $\alpha=\min \left(\mu_{1}(y, t), \mu_{2}(y, t)\right)$, model (8) is equivalent to the form of Tchebycheff model (see [7,44], which is equivalent to the following model.

\section{$\operatorname{Max} \alpha$}

subject to

$$
\begin{aligned}
& \mu_{1}(y, t) \geq \alpha, \mu_{2}(y, t) \geq \alpha, \quad \alpha \in[0,1] \text { and } \\
& (y, t) \in M=\left\{\left(y_{1}, y_{2}, t\right) \mid g_{l}(y, t) \leq 0, l=1,2, \ldots, q+m_{1}+m_{2}\right\}
\end{aligned}
$$

where $\alpha$ is the satisfactory level for both criteria of the shortest distance from the PIS and the farthest distance from the NIS. It is well known that if the optimal solution of (16) is the vector $\left(\alpha,(y, t)^{u^{*}}\right)$, then $(y, t)^{u^{*}}$ is the maximizing solution of model (8) and a satisfactory solution of the ULDM problem.

As discussed previously, the basic concept of the bi-level programming technique is that the ULDM sets his goals and/or decisions with possible tolerances which are described by membership functions of fuzzy set theory. According to this concept, let $t_{K}^{L}$ and $t_{K}^{R}, k=1,2, \ldots, n_{1}$, be the maximum acceptable negative and positive tolerance (relaxation) values on the decision vector considered by the ULDM, $(y, t)_{1}^{u^{*}}=\left((y, t)_{11}^{u^{*}},(y, t)_{12}^{u^{*}}, \ldots,(y, t)_{1 n_{1}}^{u^{*}}\right)$. The tolerances $t_{K}^{L}$ and $t_{K}^{R}$ are not necessarily the same. The tolerances give the lower level decision makers an extent feasible region to search for the satisfactory solution. If the feasible region is empty, the negative and positive tolerance must be increased to give the lower level decision makers an extent feasible region to search for the satisfactory solution $[15,19]$.

The linear membership functions (Fig.2) for each of the $n_{1}$ components of decision vector $(y, t)_{1}^{u^{*}}=\left((y, t)_{11}^{u^{*}},(y, t)_{12}^{u^{*}}, \ldots,(y, t)_{1 n_{1}}^{u^{*}}\right)$ controlled by the ULDM can be formulated as: 


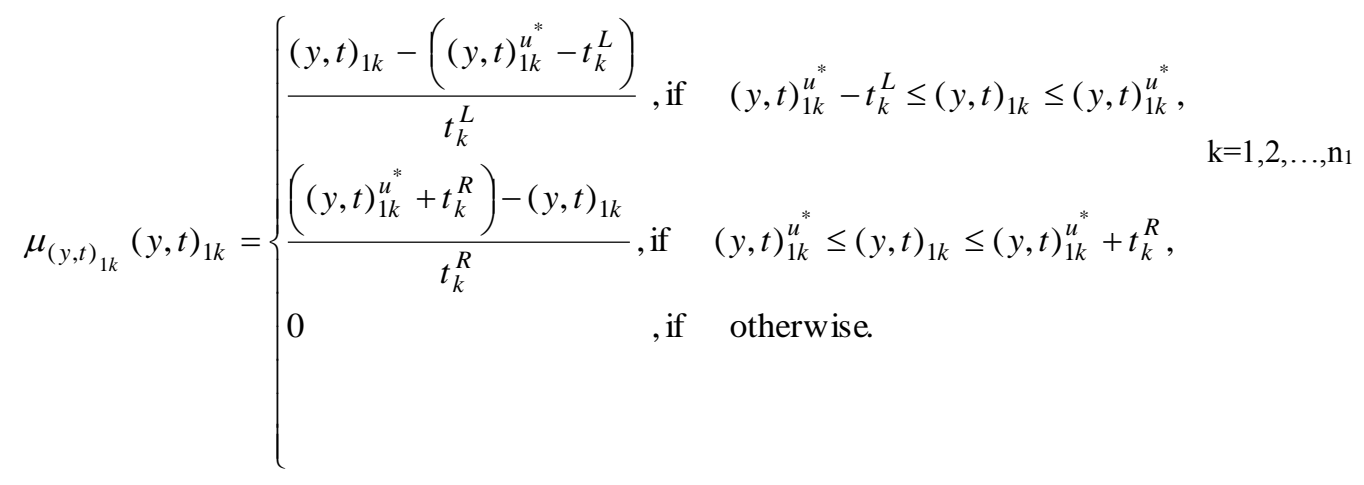

It may be noted that, the decision maker may desire to shift the range of $(y, t)_{1 k}$. Following Pramanik and Roy [15] and Sinha [16], this shift can be achieved.

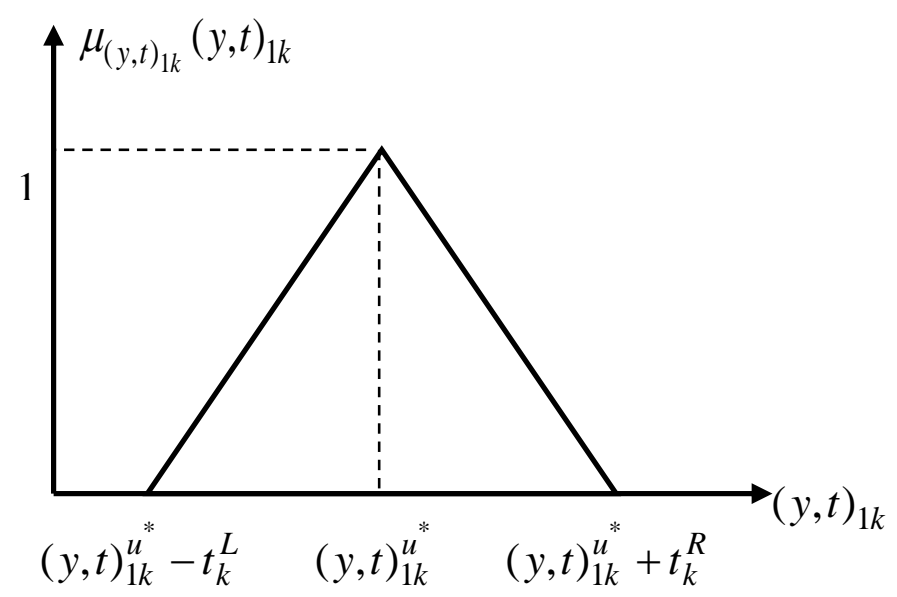

Fig. 2. The membership function of the decision variable $(y, t)_{1 k}$

\subsection{The Proposed TOPSIS Approach for BL-NMODM Problems}

In order to obtain a compromise solution (satisfactory solution) to the BL -NMODM problems using the TOPSIS approach, the distance family of (4) to represent the distance function from the positive ideal solution, $d_{p}^{P I S}$, and the distance function from the negative ideal solution, $d_{p}^{N I S^{B}}$, can be proposed in this paper for the objective functions of the upper and lower levels as follows:

$d_{p}^{P I S^{B}}(y, t)=\left\{\sum_{j=1}^{m_{1}} \lambda_{j}^{p}\left[\frac{f_{1 j}^{*}-f_{1 j}(y, t)}{f_{1 j}^{*}-f_{1 j}^{-}}\right]^{p}+\sum_{i=1}^{m_{2}} \lambda_{m_{1}+i}^{p}\left[\frac{f_{2 i}^{*}-f_{2 i}(y, t)}{f_{2 i}^{*}-f_{2 i}^{-}}\right]^{p}\right\}^{1 / p}$

and

$d_{p}^{N I S^{B}}(y, t)=\left\{\sum_{j=1}^{m_{1}} \lambda_{j}^{p}\left[\frac{f_{1 j}(y, t)-f_{1 j}^{-}}{f_{1 j}^{*}-f_{1 j}^{-}}\right]^{p}+\sum_{i=1}^{m_{2}} \lambda_{m_{1}+i}^{p}\left[\frac{f_{2 i}(y, t)-f_{2 i}^{-}}{f_{2 i}^{*}-f_{2 i}^{-}}\right]^{p}\right\}^{1 / p}$ 
where $\lambda_{k}, k=1,2, \ldots, m_{1}+m_{2}$ are the relative importance (weights) of objectives in both levels $f_{i j}^{*}=\max _{M} f_{i j}(y, t), f_{i j}^{-}=\min _{M} f_{i j}(y, t), i=1,2, j=1,2, \ldots, m_{i}, \quad$ and $\quad p=1,2, \ldots, \infty$. Let $F^{*}=\left(f_{11}^{*}, f_{12}^{*}, \ldots, f_{1 m_{1}}^{*}, f_{21}^{*}, f_{22}^{*}, \ldots, f_{2 m_{2}}^{*}\right)$, the individual positive ideal solutions for both levels, and $F^{-}=\left(f_{11}^{-}, f_{12}^{-}, \ldots, f_{1 m_{1}}^{-}, f_{21}^{-}, f_{22}^{-}, \ldots, f_{2 m_{2}}^{-}\right)$, the individual negative ideal solutions for both levels. Similarly, for the special case of $p=\infty$, see [5,44] for the general form of the distance functions that can be applied to the proposed TOPSIS approach for solving BL-NMODM problems.

In order to obtain a compromise solution, we transfer problem $\left(1^{\prime}\right)$ into the following bi-objective problem with two commensurable (but often conflicting) objectives as [2,3,4,5,7]:

$$
\begin{aligned}
& \operatorname{Min} d_{p}^{P I S^{B}}(y, t) \\
& \operatorname{Max} d_{p}^{N I S^{B}}(y, t) \\
& \text { subject to } \\
& (y, t) \in M=\left\{\left(y_{1}, y_{2}, t\right) \mid g_{l}(y, t) \leq 0, l=1,2, \ldots, q+m_{1}+m_{2}\right\}
\end{aligned}
$$

where $p=1,2, \ldots, \infty$.

Since these two objectives are usually conflicting to each other, it is possible to simultaneously obtain their individual optima. Thus, we can use membership functions to represent these individual optima. Assume that the membership functions $\left(\mu_{3}(y, t)\right.$ and $\left.\mu_{4}(y, t)\right)$ of two the objective functions are linear between $\left(d_{p}^{B}\right)^{*}$ and $\left(d_{p}^{B}\right)^{-}$, they take the following form:

$$
\begin{aligned}
& \left(d_{p}^{P I S^{B}}\right)^{*}=\min _{M} d_{p}^{P I S^{B}}(y, t) \text { and the solution is }(y, t)^{P I S} \\
& \left(d_{p}^{N I S^{B}}\right)^{*}=\max _{M} d_{p}^{N I S^{B}}(y, t) \text { and the solution is }(y, t)^{N I S} \\
& \left(d_{p}^{P I S^{B}}\right)^{-}=d_{p}^{P I S^{B}}(y, t)^{N I S} \text { or }\left(d_{p}^{P I S^{B}}\right)^{-}=\max _{M} d_{p}^{P I S^{B}}(y, t) \text { and } \\
& \left(d_{p}^{N I S^{B}}\right)^{-}=d_{p}^{N I S^{B}}(y, t)^{P I S} \text { or }\left(d_{p}^{N I S^{B}}\right)^{*}=\min _{M} d_{p}^{N I S^{B}}(y, t)
\end{aligned}
$$

Also, assume that $d_{p}^{B^{*}}=\left(\left(d_{p}^{P I S^{B}}\right)^{*},\left(d_{p}^{N I S^{B}}\right)^{*}\right)$ and $d_{p}^{B^{-}}=\left(\left(d_{p}^{P I S^{B}}\right)^{-},\left(d_{p}^{N I S^{B}}\right)^{-}\right)$. Then, based on the preference concept, we assign a larger degree to the one with shorter distance from the PIS for $\mu_{3}(y, t) \equiv \mu_{d_{p}^{P I S}{ }^{B}}(y, t)$ and assign a larger degree to the one with farther distance from NIS for $\mu_{4}(y, t) \equiv \mu_{d_{p}^{N I S} B}(y, t)$. Therefore, as shown in Fig.1, $\mu_{3}(y, t)$ and $\mu_{4}(y, t)$ can be obtained as follows: 


$$
\begin{aligned}
\mu_{3}(y, t)=\left\{\begin{array}{lll}
1 & \text {,if } & d_{p}^{P I S^{B}}(y, t)<\left(d_{p}^{P I S^{B}}\right)^{*}, \\
1-\frac{d_{p}^{P I S^{B}}(y, t)-\left(d_{p}^{P I S^{B}}\right)^{*}}{\left(d_{p}^{P I S^{B}}\right)^{-}-\left(d_{p}^{P I S^{B}}\right)^{*}} & \text {,if } & \left(d_{p}^{P I S^{B}}\right)^{*} \leq d_{p}^{P I S^{B}}(y, t) \leq\left(d_{p}^{P I S^{B}}\right)^{-}, \\
0 & \text {,if } \quad\left(d_{p}^{P I S^{B}}\right)^{-}<d_{p}^{P I S^{B}}(y, t) . \\
1 & \text {,if } \quad d_{p}^{N I S^{B}}(y, t) \geq\left(d_{p}^{N I S^{B}}\right)^{*}, \\
1-\frac{\left(d_{p}^{N I S^{B}}\right)^{*}-d_{p}^{N I S^{B}}(y, t)}{\left(d_{p}^{N I S^{B}}\right)^{*}-\left(d_{p}^{N I S^{B}}\right)^{-}} & \text {,if } \quad\left(d_{p}^{N I S^{B}}\right)^{-} \leq d_{p}^{N I S^{B}}(y, t) \leq\left(d_{p}^{N I S^{B}}\right)^{*}, \\
0 \quad \text { if } & d_{p}^{N I S^{B}}(y, t)<\left(d_{p}^{N I S^{B}}\right)^{-} .
\end{array}\right.
\end{aligned}
$$

Applying the max-min decision model, which is proposed by Bellmann and Zadeh [41] and extended by Zimmermann [42,43], the compromise solution $(y, t)$ of model (20) can be resolved and obtained by solving the following problem:

$$
\mu_{D}(y, t)=\max _{M}\left\{\min \left(\mu_{3}(y, t), \mu_{4}(y, t)\right)\right\}
$$

where $(y, t)_{1}^{*}=\left((y, t)_{11}^{*},(y, t)_{12}^{*}, \ldots,(y, t)_{1 n_{1}}^{*}\right),(y, t)_{2}^{*}=\left((y, t)_{21}^{*},(y, t)_{22}^{*}, \ldots,(y, t)_{2 n_{2}}^{*}\right)$.

If $\delta=\min \left(\mu_{3}(y, t), \mu_{4}(y, t)\right)$, the model (20) is equivalent to the form of Tchebycheff model [2,3,4,5,7,43,45,46], which is equivalent to the following model:

\section{$\operatorname{Max} \delta$}

subject to

$$
\mu_{3}(y, t) \geq \delta, \mu_{4}(y, t) \geq \delta, \quad \delta \in[0,1] \text { and }
$$

$$
(y, t) \in M=\left\{\left(y_{1}, y_{2}, t\right) \mid g_{l}(y, t) \leq 0, l=1,2, \ldots, q+m_{1}+m_{2}\right\}
$$

where $\delta$ is the satisfactory level for both criteria of the shortest distance from the PIS and the farthest distance from the NIS. It is well known that if the optimal solution of (28) is the vector $\left(\delta, y^{*}, t^{*}\right)$, then $\left(y^{*}, t^{*}\right)$ is the maximizing solution of model (20).

Finally, as discussed in section 5.1, in order to generate the satisfactory solution of the BL-NMODM problem, $(y, t)^{*}$, the final proposed model that includes the membership functions (17) for the upper level decision variables vector, $(y, t)_{1}^{u^{*}}=\left((y, t)_{11}^{u^{*}},(y, t)_{12}^{u^{*}}, \ldots,(y, t)_{1 n_{1}}^{u^{*}}\right)$, is presented, in this paper, as:

\section{$\operatorname{Max} \delta$}

subject to 


$$
\begin{aligned}
& 1-\frac{d_{p}^{P I S^{B}}(y, t)-\left(d_{p}^{P I S^{B}}\right)^{*}}{\left(d_{p}^{P I S}\right)-\left(d_{p}^{P I S^{B}}\right)^{*}} \geq \delta, \\
& 1-\frac{\left(d_{p}^{N I S^{B}}\right)^{*}-d_{p}^{N I S^{B}}(y, t)}{\left(d_{p}^{N I S^{B}}\right)^{*}-\left(d_{p}^{N I S^{B}}\right)^{-}} \geq \delta, \\
& \frac{(y, t)_{1 k}-\left((y, t)_{1 k}^{u^{*}}-t_{k}^{L}\right)^{*}}{t_{k}^{L}} \delta, \quad k=1,2, \ldots, n_{1}, \\
& \frac{\left((y, t)_{1 k}^{u^{*}}+t_{k}^{R}\right)-(y, t)_{1 k}}{t_{k}^{R}} \geq \delta, \quad k=1,2, \ldots, n_{1},
\end{aligned}
$$

$(y, t) \in M=\left\{\left(y_{1}, y_{2}, t\right) \mid g_{l}(y, t) \leq 0, l=1,2, \ldots, q+m_{1}+m_{2}\right\}$ and $\delta \in[0,1]$

\section{The TOPSIS Algorithm for BL - NMOSM Problems}

The TOPSIS model (29) provides a satisfactory decision for the two DMs at the two levels. Following the above discussion, the algorithm for the proposed TOPSIS approach, in this paper, for solving BL-NMODM problems is given as follows:

Step0. Use the transformation method $\left(\mathrm{T}_{1}\right)$ in sec. 2 to transform BL-NFMOM problems into BL- NMODM problems.

Step1. Calculate the individual minimum and maximum values of all the objective functions in the two levels under the set of constraints M.

Step2. Construct the PIS payoff table of the ULDM problem (7) and obtain $F^{u^{*}}=\left(f_{11}^{*}, f_{12}^{*}, \ldots, f_{1 m_{1}}^{*}\right)$, the individual positive ideal solutions.

Step3. Construct the NIS payoff table of the ULDM problem (7) and obtain $F^{u^{-}}=\left(f_{11}^{-}, f_{12}^{-}, \ldots, f_{1 m_{1}}^{-}\right)$, the individual negative ideal solutions.

Step4. Use Eq. (9) to construct $d_{p}^{P I S^{u}}(y, t)$ and $d_{p}^{N I S^{u}}(y, t)$.

Step5. Ask the DM to select $p,\{p=1,2, \ldots, \infty\}$.

Step6. Construct the payoff table of problem (8) and obtain $d_{p}^{u^{*}}$ and $d_{p}^{u^{-}}$.

Step7. Elicit the membership functions $\mu_{d_{p}^{P I S^{u}}}(y, t)$ and $\mu_{d_{p}^{N I S^{u}}}(y, t)$.

Step8. Formulate the model (16) for the ULDM problem.

Step9. Solve model (16) to get $(y, t)^{u^{*}}=\left((y, t)_{11}^{u^{*}},(y, t)_{12}^{u^{*}}, \ldots,(y, t)^{u^{*}}\right)$.

Step10. Set the maximum negative and positive tolerance values on the 
decision vector $(y, t)^{u^{*}}=\left((y, t)_{11}^{u^{*}},(y, t)_{12}^{u^{*}}, \ldots,(y, t)_{1 n_{1}}^{u^{*}}\right), t_{K}^{L}$ and $t_{k}^{R}, k=1,2, \ldots, n_{1}$.

Step11. Construct the PIS payoff table of the BL-NMODM problem and obtain $F^{*}=\left(f_{11}^{*}, f_{12}^{*}, \ldots, f_{1 m_{1}}^{*}, f_{21}^{*}, f_{22}^{*}, \ldots, f_{2 m_{2}}^{*}\right)$, the individual positive ideal solutions for both levels.

Step12. Construct the NIS payoff table of the BL-NMODM problem and
obtain $F^{-}=\left(f_{11}^{-}, f_{12}^{-}, \ldots, f_{1 m_{1}}^{-}, f_{21}^{-}, f_{22}^{-}, \ldots, f_{2 m_{2}}^{-}\right)$, the individual negative ideal solutions for both levels.

Step13. Use Eqs. (18) and (19) to construct $d_{p}^{P I S}(y, t)$ and $d_{p}^{N I S}(y, t)$, respectively.

Step14. Construct the payoff table of problem (20) and obtain $d_{p}^{B^{*}}$ and $d_{p}^{B^{-}}$

Step15. Elicit the membership functions $\mu_{d_{p}^{P I S} B}(y, t)$ and $\mu_{d_{p}^{N I S^{B}}}(y, t)$.

Step16. Elicit the membership functions $\mu_{(y, t)_{1 k}}(y, t)_{1 k}, k=1,2, \ldots, n_{1}$.

Step17. Formulate the model (29) for the BL-NMODM problem.

Step18. Solve model (29) to get $(y, t)^{*}=\left(y_{1}^{*}, y_{2}^{*}, t^{*}\right)$.

Step19. If the DM is satisfied with the candidate solution in step 18, go to step 20, or else go to step 21 .

Step20. Satisfactory solution is $(y, t)^{*}=\left(y_{1}^{*}, y_{2}^{*}, t^{*}\right)$ to the BL-NMODM problem.

Step21. Modify the maximum negative and positive tolerance values on the decision vector $(y, t)_{1}^{u^{*}}=\left((y, t)_{11}^{u^{*}},(y, t)_{1_{2}}^{u^{*}}, \ldots,(y, t)_{1 n_{1}}^{u^{*}}\right), t_{K}^{L}$ and $t_{k}^{R}, k=1,2, \ldots, n_{1}$, go to step 16.

The solution procedure is straightforward and illustrated via the numerical example in the following section.

\section{Illustrative Numerical Example}

The following numerical example is considered to illustrate the proposed fuzzy TOPSIS algorithm for solving BL-FMODM problems

[Upper Level]

$\underset{x_{1}}{\operatorname{Min}}\left(F_{11}=\frac{x_{1}^{2}-x_{2}^{2}}{x_{1}^{2}+x_{2}^{2}+2}, F_{12}=\frac{\left(x_{1}-2\right)^{2}-x_{2}^{2}}{\left(x_{2}-1\right)^{2}+5}\right)$

where $x_{2}$ solves

[Lower Level]

$\operatorname{Min}_{x_{2}}\left(F_{21}=\frac{\left(x_{1}-1\right)^{2}+\left(x_{2}+3\right)^{2}}{x_{1}^{2}+x_{2}+10}, F_{22}=\frac{8 x_{1}^{2}-9 x_{2}^{2}-4}{x_{1}^{2}+x_{2}^{2}+8}, F_{23}=8 x_{1}^{2}+x_{1}-\left(x_{2}-2\right)^{2}\right)$ (31)

subject to

$$
x=\left(x_{1}, x_{2}\right) \in G=\left\{\left(x_{1}, x_{2}\right) \mid x_{1}+x_{2} \leq 10,-5 x_{1}+3 x_{2} \leq 15, x_{1}, x_{2} \geq 0\right\}(32)
$$

Using the transformation method $\left(\mathrm{T}_{1}\right)$, the problem (30-32) is equivalent to the following BL-NMODM problem as:

[Upper Level] 
$\underset{y_{1}}{\operatorname{Min}}\left(f_{11}=y_{1}^{2}-y_{2}^{2}, f_{12}=\left(y_{1}-2 t\right)^{2}-y_{2} t\right)$

where $y_{2}$ solves

[Lower Level]

$\underset{y_{2}}{\operatorname{Min}}\left(f_{21}=\left(y_{1}-t\right)^{2}+\left(y_{2}+3 t\right)^{2}, f_{22}=8 y_{1}^{2}-9 y_{2}^{2}-4 t^{2}, f_{23}=8 y_{1}^{2}+y_{1} t-\left(y_{2}-2 t\right)^{2}\right)(31)^{\prime}$

subject to

$$
\begin{aligned}
&(y, t) \in M=\left\{\left(y_{1}, y_{2}, t\right) \mid y_{1}^{2}+y_{2}^{2}+2 t^{2} \leq 1,\left(y_{2}-t\right)^{2}+5 t^{2} \leq 1,\right. \\
& y_{1}^{-2} t^{3}+y_{2}+10 t \leq 1, y_{1}^{2}+y_{2}^{2}+8 t^{2} \leq 1, t^{2} \leq 1, \\
&\left.y_{1}+y_{2}-10 t \leq 0,-5 y_{1}+3 y_{2}-15 t \leq 0, y_{1}, y_{2} \geq 0, t>0\right\} .
\end{aligned}
$$

Table 1 summarizes minimum and maximum individual optimal solutions, of all objectives functions for the two levels of the BLNMODM problem, subjected to given constraints $\mathrm{M}$.

\section{Table 1}

Minimum and maximum individual optimal solutions.

\begin{tabular}{cccccc}
\hline & $f_{11}$ & $f_{12}$ & $f_{21}$ & $f_{22}$ & $f_{23}$ \\
\hline $\operatorname{Min}_{M} f_{i j}$ & -0.184 & -0.024 & 0 & -1.682 & -0.041 \\
$\operatorname{Max}_{M} f_{i j}$ & 0.54 & 0.593 & 0.842 & & 7.37 \\
\hline
\end{tabular}

\section{Upper-Level NMODM problem:}

We first obtain PIS and NIS payoff tables for the upper-level NMODM problem (Tables 2 and 3):

$$
\underset{y_{1}}{\operatorname{Min}}\left(f_{11}(y, t)=y_{1}^{2}-y_{2}^{2}, f_{12}(y, t)=\left(y_{1}-2 t\right)^{2}-y_{2} t\right)
$$

subject to

$$
(y, t)=\left(y_{1}, y_{2}, t\right) \in M
$$

Table 2

PIS payoff table of problem (33) and (34).

\begin{tabular}{llcccc}
\hline & $f_{11}$ & $f_{12}$ & $y_{1}$ & $y_{2}$ & $t$ \\
\hline $\operatorname{Min}_{M} f_{11}$ & $-0.184^{*}$ & 0.718 & 0.102 & 0.441 & 0.54 \\
\hline $\begin{array}{l}M i n \\
M\end{array}$ & & & & \\
\hline$F^{u}$ & -0.178 & $-0.024^{*}$ & 0.112 & 0.437 & \\
\end{tabular}

Table 3

NIS payoff table of problem (33) and (34).

\begin{tabular}{cccccc}
\hline & $f_{11}$ & $f_{21}$ & $y_{1}$ & $y_{2}$ & $t$ \\
\hline $\operatorname{Max}_{11}$ & $0.926^{-}$ & 0.58 & 0.962 & 0 & 0.1
\end{tabular}




\begin{tabular}{cccccc}
$\operatorname{Maxf}_{12}$ & 0.925 & $0.593^{-}$ & 0.962 & 0 & 0.096 \\
\hline
\end{tabular}

$F^{u^{-}}=\left(f_{11}^{-}, f_{12}^{-}\right)=(0.926,0.593)$

Assume that $\lambda_{1}=\lambda_{2}=0.5$, the equations for $d_{p}^{P I S^{u}}$ and $d_{p}^{N I S^{u}}(y, t)$ when $\mathrm{p}=2$ are:

$$
\begin{aligned}
& F_{1}^{u}=d_{2}^{P I S^{u}}(y, t)=\left\{\lambda_{1}^{2}\left[\frac{\left(y_{1}^{2}-y_{2}^{2}\right)+0.184}{0.926+0.184}\right]^{2}+\lambda_{2}^{2}\left[\frac{\left(y_{1}-2 t\right)^{2}-y_{2} t+0.024}{0.593+0.024}\right]^{2}\right]^{1 / 2} \\
& F_{1}^{u}=d_{2}^{P I S^{u}}(y, t)=\left\{0.203\left[\left(y_{1}^{2}-y_{2}\right)^{2}+0.184\right]^{2}+0.657\left[\left(y_{1}-2 t\right)^{2}-y_{2} t+0.024\right]^{2}\right\}^{1 / 2} \\
& F_{2}^{u}=d_{2}^{N I S^{u}}(y, t)=\left\{\lambda_{1}^{2}\left[\frac{0.926-\left(y_{1}^{2}-y_{2}^{2}\right)}{0.926+0.184}\right]^{2}+\lambda_{2}^{2}\left[\frac{0.593-\left(y_{1}-2 t\right)^{2}+y_{2} t}{0.593+0.024}\right]^{2}\right\}^{1 / 2} \\
& F_{2}^{u}=d_{2}^{N I S^{u}}(y, t)=\left\{0.203\left[0.926-\left(y_{1}^{2}-y_{2}^{2}\right)\right]^{2}+0.657\left[0.593-\left(y_{1}-2 t\right)^{2}+y_{2} t\right]^{2}\right\}^{1 / 2}
\end{aligned}
$$

\begin{tabular}{|c|c|c|c|c|c|}
\hline & $F_{1}^{u}$ & $F_{2}^{u}$ & $y_{1}$ & $y_{2}$ & $t$ \\
\hline$\underset{M}{\operatorname{Max}} F_{1}^{u}$ & $0.707^{*}$ & $0^{-}$ & 0.962 & 0 & 0.096 \\
\hline $\operatorname{Min}_{M} F_{2}^{u}$ & $0.707^{-}$ & $0^{*}$ & 0.962 & 0 & 0.096 \\
\hline
\end{tabular}

Next, to formulate model (16) the payoff table of (8) is shown in Table4:

\section{Table 4}

The payoff table of (8) when $p=2$.

Also, $\operatorname{Min} F_{1}^{u}=0$ at $(0.103,0.441,0.054)$ and $\operatorname{Max}_{2}^{u}=0.707$ at $(0.102,0.441,0.054)$. Thus, we have $d_{2}^{u^{*}}=(0.707,0)$ and $d_{2}^{u^{-}}=(0,0.707)$ (as proposed in this paper). Therefore, the membership functions $\mu_{F_{1}}(y, t)$ and $\mu_{F_{2}}(y, t)$ can be obtained as:

$$
\begin{aligned}
& \mu_{F_{1}^{u}}(y, t)=1-\frac{F_{1}^{u}-0.707}{0-0.707}=0.0003+1.414 F_{1}^{u} \\
& \mu_{F_{2}^{u}}(y, t)=1-\frac{0.707-F_{2}^{u}}{0-0.707}=2-1.414 F_{2}^{u}
\end{aligned}
$$

And then, the equivalent TOPSIS formulation for the ULDM problem is obtained as:

\section{$\operatorname{Max} \alpha$}


subject to

$$
\begin{aligned}
& 0.0003+1.414 F_{1}^{u} \geq \alpha, 2-1.414 F_{2}^{u} \geq \alpha, \\
& \alpha \in[0,1], \text { and }(\mathrm{y}, \mathrm{t})=\left(\mathrm{y}_{1}, y_{2}, t\right) \in M .
\end{aligned}
$$

The maximum satisfactory level $\alpha=0.707$ is achieved for the solution $y_{1}=0.962, y_{2}=0$ and $t=0.096$, and $\left(f_{11}, f_{12}\right)=(0.925,0.593)$. Let the upper level DM decide $y_{1}^{u^{*}}=0.962$ with positive tolerance $t^{R}=0.5$ (one sided membership function [15,19].

\section{The BL-NMODM problem:}

We first obtain PIS and NIS payoff tables for the lower-level NMODM problem (Tables 5 and 6):

$\operatorname{Min}\left(f_{21}=\left(y_{1}-t\right)^{2}+\left(y_{2}+3 t\right)^{2}, f_{22}=8 y_{1}^{2}-9 y_{2}-4 t^{2}, f_{23}=8 y_{1}^{2}+y_{1} t-\left(y_{2}-2 t\right)^{2}\right)(35)$ $y_{2}$

subject to

$$
(y, t)=\left(y_{1}, y_{2}, t\right) \in M .
$$

\begin{tabular}{|c|c|c|c|c|c|c|}
\hline & $f_{21}$ & $f_{22}$ & $f_{23}$ & $y_{1}$ & $y_{2}$ & $t$ \\
\hline $\operatorname{Min}_{M} f_{21}$ & $0^{*}$ & 0 & 0 & 0 & 0 & 0 \\
\hline $\operatorname{Min}_{M} f_{22}$ & 0.382 & $-1.682^{*}$ & -0.11 & 0.102 & 0.441 & 0.045 \\
\hline $\operatorname{Min}_{M} f_{23}$ & 0.295 & -1.235 & $-0.041^{*}$ & 0.054 & 0.372 & 0.057 \\
\hline
\end{tabular}

Table 5

PIS payoff table of problem (35) and (36).

\begin{tabular}{|c|c|c|c|c|c|c|}
\hline & $f_{21}$ & $f_{22}$ & $f_{23}$ & $y_{1}$ & $y_{2}$ & $t$ \\
\hline $\operatorname{Max}_{M} f_{21}$ & $0.842^{-}$ & 7.33 & 7.436 & 0.96 & 0.019 & 0.098 \\
\hline $\operatorname{Max}_{M} f_{22}$ & 0.833 & $7.37^{-}$ & 7.459 & 0.962 & 0 & 0.096 \\
\hline $\operatorname{Max}_{M} f_{23}$ & 0.833 & 7.37 & $7.463^{-}$ & 0.962 & 0 & 0.096 \\
\hline
\end{tabular}

\section{Table 6}

NIS payoff table of problem (35) and (36).

$F^{-} L=\left(F_{21}^{-}, F_{22}^{-}, F_{23}^{-}\right)=(0.842,7.37,7.463)$

Assume that $\lambda_{1}=\lambda_{2}=\lambda_{3}=1 / 3$, the equations for $d_{p}^{P I S^{L}}(y, t)$ and $d_{p}^{N I S^{L}}(y, t)$ when $p=2$ are:

$F_{3}^{L}=d_{2}^{P I S^{L}}(y, t)=\left\{\begin{array}{l}0.157\left(\left(y_{1}-t\right)^{2}+\left(y_{2}+3 t\right)^{2}\right)^{2}+0.001\left(8 y_{1}^{2}-9 y_{2}^{2}-4 t^{2}+1.682\right)^{2} \\ +0.002\left(8 y_{1}^{2}+y_{1} t-\left(y_{2}-2 t\right)^{2}+0.041\right)^{2}\end{array}\right\}^{1 / 2}$ 
$F_{4}^{L}=d_{2}^{N I S^{L}}(y, t)=\left\{\begin{array}{l}0.157\left(0.842-\left(y_{1}-t\right)^{2}-\left(y_{2}+3 t\right)^{2}\right)^{2}+0.001\left(7.37-\left(8 y_{1}^{2}-9 y_{2}-4 t^{2}\right)\right)^{2} \\ +0.002\left(7.463-\left(8 y_{1}^{2}+y_{1} t-\left(y_{2}-2 t\right)^{2}\right)\right)^{2}\end{array}\right\}^{1 / 2}$

Next, to formulate model (29) the payoff table of (20) is shown in Table7.

Table 7

The payoff table of (20), when $\mathrm{p}=2$.

\begin{tabular}{|c|c|c|c|c|c|}
\hline & $P_{1}^{B}$ & $P_{2}^{B}$ & $y_{1}$ & $y_{2}$ & $t$ \\
\hline $\operatorname{Max} F_{3}^{L}$ & 0.552 & 0.559 & 0.96 & 0.019 & 0.098 \\
\hline $\operatorname{Min} F_{4}^{L}$ & 0.05 & 0.144 & 0.96 & 0.019 & 0.98 \\
\hline
\end{tabular}

Thus, the membership functions $\mu_{F_{3}{ }^{L}}(y, t)$ and $\mu_{F_{4}{ }^{L}}(y, t)$ can be obtained as:

$$
\begin{aligned}
& \mu_{F_{3}^{L}}(y, t)=1-\frac{F_{3}^{L}-0.552}{0.05-0.552}=1.992 F_{3}^{L}-0.1 \\
& \mu_{F_{4}^{L}}(y, t)=1-\frac{0.552-F_{4}^{L}}{0.144-0.554}=2.351-2.439 F_{4}^{L}
\end{aligned}
$$

Finally, the equivalent TOPSIS formulation for BL-NMODM problem is obtained as:

$$
\begin{aligned}
& 1.992 F_{3}^{L}-0.1 \geq \delta, \\
& 2.351-2.439 F_{4}^{L} \geq \delta, \\
& 2.165 y_{1}-1.082 \delta \geq 1, \\
& 0.684 y_{1}+0.342 \delta \leq 1, \\
& 0 \leq \delta \leq 1, \text { and }(y, t) \in M .
\end{aligned}
$$

The maximum overall satisfactory level of the BL-NMODM problem $\delta=0.999$ is achieved for the solution $y_{1}=0.961, y_{2}=0.013$ and $t=0.097$. By using the transformation $\left(\mathrm{T}_{1}\right)$, then the compromise solution is $x_{1}=9.907$ and $x_{2}=0.134, \quad$ with $\quad$ objective $\quad$ function $\quad$ values $f_{11}=0.98, f_{12}=$ 10.85, $f_{21}=8.79, f_{22}=7.36$ and $f_{23}=791.61 \quad$ and $\quad$ with $\quad$ membership function values $\mu_{F_{1}^{u}}=0.996, \mu_{F_{2}^{u}}=1, \mu_{F_{3}{ }^{L}}=0.991$ and $\mu_{F_{4}{ }^{L}}=0.88$, respectively.

\section{Conclusion}

The technique for order preference by similarity to ideal solution (TOPSIS) is considered the advantage approach for NMODM problems. In this paper, a TOPSIS approach is proposed for solving bi-level fractional non-linear multi-objective decision making (BL-FNMODM) problems. A compromise solution (satisfactory solution) can be obtained to the BL- FNMODM problems by using the transformation $\left(\mathrm{T}_{1}\right)$ to convert BL- FNMODM problem into BL-NMODM problem and using the concept of TOPSIS approach which represents the family distance function from the positive ideal solution (PIS) and the distance function from the negative ideal 
solution (NIS) in the proposed formula in the objective functions of both the upper and lower levels. Then, the bi-Level problem can be solved by using membership functions of fuzzy set theory to represent the satisfaction level for both criteria and obtain TOPSIS's compromise solution by a second -order compromise. The max-min operator is then considered as a suitable one to resolve the conflict between the new criteria (the shortest distance from PIS and the longest distance from NIS). Finally, an illustrative numerical example is given to demonstrate the proposed TOPSIS approach for BL- FNMODM problems.

\section{References}

[1] C.L. Hwang, K. Yoon., Multiple Attribute Decision Making: Methods and Applications., Springer-Verlag, Heidelberg,(1981).

[2] M.A. Abo-Sinna., Extensions of the TOPSIS for Multi-Objective Dynamic Programming Problems under Fuzziness., Adv. Model. Anal. (AMSE Press, France)., 43 (4), 1-24, (2000).

[3] M. A. Abo-Sinna, A. H. Amer., Extensions of TOPSIS for Multi Objective Large Secale Nonlinear Programming Problems., Appl. Math- Comput.., 162, $243-256,(2000)$.

[4] M.A. Sbo-Sinna, A.H. Amer, A.S. Ibrahim., Extensions of TOPSIS for Large Scale Multi-Objective Non-Linear Programming problems with Block Angular Structure., Appl. Math. Model.., 32, 292-302, (2008).

[5] Y. J. Lai, T.J. Liu, C.L. Hwang., TOPSIS for MODM., Euro. J. Oper. Res.., 76, 486-500, (1994).

[6] M. Zeleny., Multiple Criteria Decision Making., McGraw, Hill Book Company, New York, (1982).

[7] I.A. Baky, M.A. Abo-Sinna., TOPSIS for Bi-Level MODM Problems., Applied Mathematical Modelling., 37, 1004-1015,(2013).

[8] H. Deng, C.H. Yeh, R.J. Willis., Inter-Company Comparison using Modified TOPSIS with Objective Weights., Comput. Oper. Res.., 17, 963-973,(2000).

[9] C.T. Chen., Extensions of the TOPSIS for Group Decision-Making under Fuzzy Environment., Fuzzy Sets and Systems., 114, 1-9, (2000).

[10] L.N. Vicent, and P.H. Calamai., Bi-level and Multi-level Programming., a Bibliography Review, J. Global Optim.., 5, 291-306, (1994).

[11] K. Mathur, M.C. Puri., A bi-level Bottleneck Programming Problem., European Journal of Oprational Research., 86, 337$344,(1995)$

[12] G. Anandilingam., A Mathematical Programming Model of Decentralized Multi-Level System., Journal of the Operational Research Society., 39 (11), 1021-1033, (1988).

[13] G. Anandilingam, V. Apprey., Multi-Level Programming and Conflicting Resolution., European Journalof Operational Research., 51, 233-247, (1991).

[14] S.R. Arora, R. Gupta., Interactive Fuzzy Goal Programming Approach for Bi-Level Programming Problem., European Journal of Operational Research., 194, 368-376, (2009).

[15] S. Pramanik,T. Kumar Roy., Fuzzy Goal Programming Approach to Multi-Level Programming problems., Euro. J. Oper. Res.., 176, 1151-1166, (2006).

[16] H.S. Shih, Y.J. Lai, E.S. Lee., Fuzzy Approach for Multi-Level Programming Problems., Computers and Operations Research., 23 (1), $773-791,(1983)$.

[17] H. S. Shih, E.S,. Lee., Compensatory Fuzzy Multiple Decision Making., Fuzzy Sets and Systems., 14, 71- 87, (2000).

[18] S. Sinha., Fuzzy Mathematical Approach to Multi-Level Programming Problems., Computers and Operations Research., 30, 1259-1268, (2003).

[19] S. Sinha., Fuzzy Programming Approach to Multi-Level Programming Problems., Fuzzy Sets and Systems., 136, 189-202, (2003).

[20] W. Candler, R. Townsley., A Linear Two Level Programming Problem., Comput. Oper Res.., 9 (1), 59-76, (1982).

[21] J. Bracken, J.E. Falk, F.A. Miercort., A Strategic Weapons Exchange Allocation Model., Oper. Res.., 25, 968-976, (1977).

[22] R.G. Cassidy, M.J.L. Kirby, W.H. Raike., Efficient Distribution of Resources through three Levels of Government., Manag. Sci.., $17,462-473,(1971)$.

[23] R.E. Bellmann., Dynamic Programming., Princeton University Press, Princeron, NJ, (1957).

[24] I.A. Baky., Solving Multi-Level Multi-Objective Linear Programming Problems through Fuzzy Goal Programming Approach., Applied Mothematical Modelling., 34,2377-2387, (2010).

[25] M.A. Abo-Sinna, I.A. Baky., Interactive Balance Space Approach for Solving Multi-Level Multi-Objective Programming Problems., Inform. Sci.., 177,3397- 3410, (2007).

[26] I.A. Baky., Fuzzy Goal Programming Algorithm for Solving Decentralized Bi-Level Multi-Objective Programming Problems., Fuzzy Sets and Systems., 160, 2701-2710, (2009). 
[27] K. Lachhwani., On Solving Multi-Level Multi-Objective Linear Fractional Programming Problems through Fuzzy Goal Programming Approach., Journal of Operational Research Society of India OPSEARCH.,51 (4), 624-637, (2014).

[28] K. Lachhwani., Modified FGP Approach for Multi-Level Mult- Objective Linear Fractional Programming Problems., Applied Mathematics and Computation., 266, 1038-1049, (2015).

[29] M.A. Abo-Sinna, I.A. Baky., Interactive Balance Space Approach for Solving Bi-Level Multi-Objective Programming Problems., AMSE- Model, Adv. BJ., 40, 43-62. France, (2006).

[30] Dr. Madhuchanda Rakshit, Mr. Suchet Kumar., A Bi-Level Quadratic- Quadratic Fractional Programming through Fuzzy Goal Programming Approach., IJMTT, 38,3,October, (2016).

[31] Deepak Bhati, Pitam Singh and Rubi Arya., A Taxonomy and Review of the Multi-Objective Fractional Programming (MOFP) Problems., Int.J. Appl. Comput. Math.., 21, October, (2016).

[32] F. Chen, G.H. Huang, Y.R. Fan and R. F.Lias., A Non-linear Fractional Programming Approach for Environmental-Economic Power Dispatch., Electrical Power and Energy Systems., 78, 463-469, (2016).

[33] Dr. Savita Mishra., Arun Bihari Verma and Indrani Dey., Quadratic Fractional Multi-Level Programming Problem Based on Fuzzy Goal Programming Approach., V.1 Special Issue, July, (2016).

[34] Nocera Maachou and Mustapha Moulai., Bi-Level Quadratic Fractional/ Quadratic Problem., Springer Int. Publishing Switzerland, (2015).

[35] K.C. Sharma and Jitendra Singh., Quadratic Fractional Optimization through FGP Approach., Int. Journal of Science and Research (IJSR)., V.3, Issues 5, May, (2014).

[36] Savita Mishra and Ajit Ghosh., Interactive Fuzzy Programming Approach to Bi-Level Quadratic Fractional Programming Problems., Ann. Oper. Res.., 143, 251-263, (2006).

[37] A. Charnes, W. Cooper., Programming with Linear Fractional Functions., Naval Research Logistic Quartely., 9(3-4), 181-186, (1962).

[38] S. Mishra. A. Ghosh., Interactive Fuzzy Programming Approach to Bi-Level Quadratic Fractional Programming Problems., Annals of Operations Research., 143, 251-263, (2006).

[39] K. Miettinen., Non-linear Multi-Objective Optimization., Kluwer Academic Publishers, (2002).

[40] P.L. YM, M. Zeleny., the Set of All Non-Dominated Solution in Linear Cases and Multi-Criteria Simplex Method., J. Math. Analy. Appl.., 49, 430-448, (1975).

[41] R.E. Bellmann, L.A. Zadeh., Decision-Making in a Fuzzy Environment, Manag., Sci.., 17, 141-164, (1970).

[42] H.J. Zimmermann., Fuzzy Programming and Linear Programming with Several Objective Functions., Fuzzy Sets Syst.., 1, 45-55, (1978).

[43] H.J. Zimmermann., Fuzzy Sets Decision Making and Expert Systems., Kluwer Academic, Boston, (1987).

[44] Y. J. Lai, C.L. Hwang., A New Approach to Some Possibility Linear Programming Problems., Fuzzy Sets Syst.., 49, 121-134, (1992). 